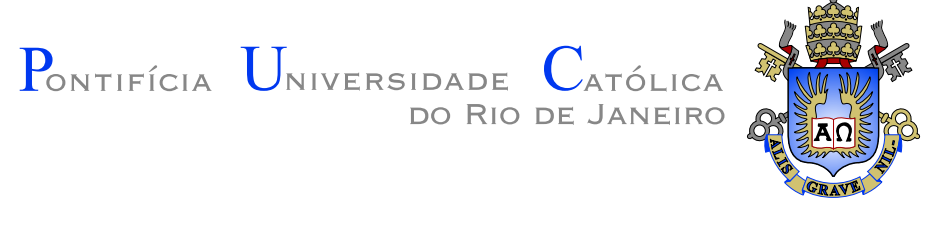

Maria Clara Schuwartz Ferreira

\title{
Hipersuperfícies Equivariantes Mínimas e com Curvatura Média Constante em $\mathbb{S}^{n}$ e $\mathbb{H}^{n}$
}

Dissertação apresentada como requisito parcial para obtenção do grau de Mestre pelo Programa de Pós-graduação em Matemática Pura do Departamento de Matemática da PUC-Rio

Orientador: Prof. Henri Anciaux 
Maria Clara Schuwartz Ferreira

\title{
Hipersuperfícies Equivariantes Mínimas e com Curvatura Média Constante em $\mathbb{S}^{n}$ e $\mathbb{H}^{n}$
}

Dissertação apresentada como requisito parcial para obtenção do grau de Mestre pelo Programa de Pós-graduação em Matemática Pura do Departamento de Matemática do Centro Técnico Científico da PUC-Rio. Aprovada pela Comissão Examinadora abaixo assinada.

\author{
Prof. Henri Anciaux \\ Orientador \\ Departamento de Matemática — PUC-Rio \\ Prof. Maria Fernanda Elbert \\ Departamento de Matemática — UFRJ \\ Prof. Ricardo Sá Earp \\ Departamento de Matemática — PUC-Rio \\ Prof. Walcy Santos \\ Departamento de Matemática — UFRJ
}

Prof. José Eugenio Leal

Coordenador Setorial do Centro Técnico Científico - PUC-Rio 
Todos os direitos reservados. É proibida a reprodução total ou parcial do trabalho sem autorização da universidade, do autor e do orientador.

Maria Clara Schuwartz Ferreira

Graduação: Bacharelado em Matemática - Universidade Federal do Espírito Santo - UFES (2002-2005).

Ficha Catalográfica

Ferreira, Maria Clara S.

Hipersuperfícies Equivariantes Mínimas e com Curvatura Média Constante em $\mathbb{S}^{n}$ e $\mathbb{H}^{n}$ / Maria Clara Schuwartz Ferreira; orientador: Henri Anciaux. - Rio de Janeiro : PUCRio, Departamento de Matemática, 2007.

v., 69 f: il. ; $29,7 \mathrm{~cm}$

1. Dissertação (mestrado) - Pontifícia Universidade Católica do Rio de Janeiro, Departamento de Matemática.

Inclui referências bibliográficas.

1. Matemática - Tese. 2. Hipersuperfícies Mínimas. 3. Hipersuperfícies com Curvatura Média Constante. 4. Geometria Equivariante. 5. Formas Espaciais. I. Anciaux, Henri. II. Pontifícia Universidade Católica do Rio de Janeiro. Departamento de Matemática. III. Título. 


\section{Agradecimentos}

Ao meu orientador Henri, que além de ter sido um excelente orientador, foi também um amigo, sempre me incentivando e acreditando no meu trabalho.

Aos membros da banca, em especial o Prof. Ricardo, que enriqueceu muito a minha dissertação com valiosas sugestões. Obrigada por sua dedicação, apoio e carinho.

Ao Carlos, que com seu conhecimento e paciência, me acompanhou nos momentos mais difíceis.

Ao Eduardo, que além da amizade, me forneceu ajuda preciosa nos problemas computacionais.

Aos funcionários, principalmente Creuza, Kátia, Orlando e Otávio, que com muita boa vontade e simpatia facilitaram o meu cotidiano na PUC.

Aos meus colegas e amigos, que tornaram esses dois anos mais alegres.

Ao Marcelo, pelo seu companheirismo e compreensão.

E especialmente, agradeço com muito carinho aos meus pais e meus irmãos, pelo grande apoio que me deram. 


\section{Resumo}

Ferreira, Maria Clara S.; Anciaux, Henri. Hipersuperfícies Equivariantes Mínimas e com Curvatura Média Constante em $\mathbb{S}^{n}$ e $\mathbb{H}^{n}$. Rio de Janeiro, 2007. 69p. Dissertação de Mestrado - Departamento de Matemática, Pontifícia Universidade Católica do Rio de Janeiro.

Neste trabalho estudamos hipersuperfícies equivariantes mínimas ou com curvatura média constante imersas em $\mathbb{S}^{n}$ e $\mathbb{H}^{n}$. Tais hipersuperfícies são construídas a partir de uma curva em $\mathbb{S}^{2}$ e em $\mathbb{H}^{2}$ respectivamente, chamada de curva geratriz. A equação da curvatura média constante reduz-se a um sistema de EDO sobre a curva geratriz, e graças à simetria do problema, podemos eliminar uma variável desse sistema. O sistema simplificado, por sua vez, admite uma integral primeira. No caso esférico, encontramos condições para obter curvas soluções fechadas, produzindo assim exemplos de hipersuperfícies compactas mínimas ou com curvatura média constante em $\mathbb{S}^{n}$. Discutimos também a questão do mergulho dessas hipersuperfícies. No caso hiperbólico, nos limitamos ao caso das hipersuperfícies mínimas; observamos que as curvas soluções não são fechadas e tratamos da questão do mergulho.

\section{Palavras-chave}

Hipersuperfícies Mínimas. Hipersuperfícies com Curvatura Média Constante. Geometria Equivariante. Formas Espaciais. 


\section{Abstract}

Ferreira, Maria Clara S.; Anciaux, Henri. Minimal and Constant Mean Curvature Equivariant Hypersurfaces in $\mathbb{S}^{n}$ and $\mathbb{H}^{n}$. Rio de Janeiro, 2007. 69p. MsC Thesis - Departament of Mathematics, Pontifícia Universidade Católica do Rio de Janeiro.

In this work we study equivariant hypersurfaces in $\mathbb{S}^{n}$ and $\mathbb{H}^{n}$ which are minimal or have constant mean curvature. These hypersurfaces are described via a curve in $\mathbb{S}^{2}$ and $\mathbb{H}^{2}$ respectively, called the generating curve. In the equivariant case, the constant mean curvature equation reduces to an ODE on the generating curve, which can be reduced by one variable using the symmetry of the problem. It then turns out that this reduced system admits a first integral. In the spherical case, we find conditions insuring closedness of the integral curves, and we deduce the existence of compact hypersurfaces which are minimal or have constant mean curvature. We also discuss the question of embeddedness of these hypersurfaces. In the hyperbolic case, we limit ourselves to the minimal case. We observe that the curves are no longer closed and again we discuss embededdness.

\section{Keywords}

Minimal Hypersurfaces. Constante Mean Curvature Hypersurfaces. Equivariant Geometry. Space Forms. 


\section{Sumário}

\begin{tabular}{lr}
\hline Lista de figuras & 8
\end{tabular}

$\begin{array}{llr}1 & \text { Preliminares } & 11\end{array}$

$\begin{array}{lll}1.1 & \text { Variedades Diferenciáveis } & 11\end{array}$

$\begin{array}{lll}1.2 & \text { Variedades Riemannianas } & 13\end{array}$

$\begin{array}{lll}1.3 & \text { Conexão Riemanniana } & 14\end{array}$

1.4 A Segunda Forma Fundamental de uma imersão de codimensão 1

\begin{tabular}{lll}
\hline 1.5 & O espaço hiperbólico 20
\end{tabular}

$\begin{array}{lll}1.6 & \text { As formas espaciais } & 21\end{array}$

2 Hipersuperfícies Equivariantes Mínimas e com Curvatura Média Constante em $\mathbb{S}^{n+1} \quad 23$

$\begin{array}{lll}2.1 & \text { Hipersuperfícies Equivariantes em } \mathbb{S}^{n+1} & 23\end{array}$

$\begin{array}{lll}2.2 & \text { O caso Mínimo } & 29\end{array}$

2.3 O Caso Curvatura Média Constante 38

3 Hipersuperfícies Equivariantes Mínimas e com Curvatura Média Cons$\begin{array}{ll}\text { tante em } \mathbb{H}^{n+1} & 53\end{array}$

$3.1 \quad$ Hipersuperfícies Equivariantes em $\mathbb{H}^{n+1} \quad 53$

3.2 O caso Mínimo 62

\begin{tabular}{ll}
\hline Referências Bibliográficas & 68
\end{tabular} 


\section{Lista de figuras}

2.1 Gráfico da função $e(\phi)=\operatorname{sen} \phi \cos ^{n-1} \phi$

2.2 Diagrama de fase esférico no caso mínimo, $n=2$

2.3 Diagrama de fase esférico no caso CMC, $H=1, n=2$

3.1 Gráfico da função $e(\phi)=\operatorname{senh} \phi \cosh ^{n-1} \phi$

3.2 Diagrama de fase hiperbólico no caso mínimo, $n=4$ 